\title{
Experimentation with tobacco during adolescence as a factor influencing treatment of smoking in adulthood. A retrospective cohort
}

\author{
Carolina Hanna Chaim', Erica Rosanna Siu", Carlos Felipe Cavalcanti Carvalho"l', Fernanda Piotto Frallonardo'v, Flavia Ismaelv,
} Arthur Guerra de Andrade ${ }^{\mathrm{VI}}$, Antonio Ventriglio ${ }^{\mathrm{VII}}$, Julio Torales ${ }^{\mathrm{VIII}}$, Dinesh Bhugra' ${ }^{\mathrm{IX}}$, João Mauricio Castaldelli-Maia ${ }^{\mathrm{x}}$

Faculdade de Medicina do ABC (FMABC), Santo André (SP), Brazil

IMD. Research Associate, Department of Psychiatry, Faculdade de Medicina da Universidade de São Paulo (FMUSP), São Paulo (SP), Brazil.

(D) orcid.org/0000-0001-8227-0423

"PhD. Research Associate, Department of Psychiatry, Faculdade de Medicina da Universidade de São Paulo (FMUSP), São Paulo (SP), Brazil.

(D) orcid.org/0000-0001-7492-7579

"'MD. Research Associate, ABC Center for Mental Health

Studies, Santo André (SP), Brazil.

(D) orcid.org/0000-0002-2269-4918

"MD. Research Associate, ABC Center for Mental Health Studies, Department of Neuroscience, Faculdade de

Medicina do $A B C$ (FMABC), Fundação ABC, Santo André

(SP), Brazil.

(D) orcid.org/0000-0001-6225-5162

vMD, PhD.Vice-President, ABC Center for Mental Health Studies, Department of Neuroscience, Faculdade de

Medicina do $A B C$ (FMABC), Fundação $A B C$, Santo André (SP), Brazil. Auxiliary Professor of Medicine, Universidade de São Caetano do Sul (USCS), São Caetano do Sul (SP), Brazil.

(D) orcid.org/0000-0002-4837-9318

"MD, PhD. Associate Professor, Department of Psychiatry, Faculdade de Medicina da Universidade de São Paulo

(FMUSP), São Paulo (SP), and Full Professor, ABC Center for Mental Health Studies, Department of Neuroscience, Faculdade de Medicina do ABC (FMABC), Fundação ABC

Santo André (SP), Brazil.

(D) orcid.org/0000-0002-6856-8212

"IIMD, PhD. Honorary Researcher, Department of Clinical and Experimental Medicine, University of Foggia, Foggia, Italy.

(iD orcid.org/0000-0002-3934-7007

"IIIMD, PhD. Assistant Professor, Department of Psychiatry, Medical School, National University of Asunción, San

Lorenzo, Central, Paraguay.

(D) orcid.org/0000-0003-3277-7036

XMA, MSC, MBBS, FRCP, FRCPsych, FFPH, PhD, FACP, FAMS. Emeritus Professor, Institute of Psychiatry, Psychology \& Neuroscience, King's College, London, United Kingdom. (iD orcid.org/0000-0002-0613-2480

×MD, PhD. Research Associate, Department of Psychiatry, Faculdade de Medicina da Universidade de São Paulo (FMUSP), São Paulo (SP), and Auxiliary Professor, ABC Center for Mental Health Studies, Department of Neuroscience, Faculdade de Medicina do ABC (FMABC), Fundação ABC Santo André (SP), Brazil.

(D) orcid.org/0000-0001-9621-2291

KEY WORDS:

Adolescent.

Tobacco.

Nicotine

Treatment outcome.

\begin{abstract}
BACKGROUND: There are still few studies on predictors of smoking cessation in Brazilian samples. Experimentation with tobacco during adolescence (ETA) may be one of the important predictors.

OBJECTIVE: This study aimed, within the context of a treatment-seeking group of subjects, to test the hypothesis that ETA negatively affects the outcome of smoking cessation during adulthood.

DESIGN AND SETTING: Retrospective (historic) cohort study conducted at a psychosocial care center in São Paulo, Brazil, between 2007 and 2010.

METHODS: Data on sociodemographics, smoking and medical profiles were obtained through self-report questionnaires that were completed at the baseline and at any follow-up appointment. Logistic regression models were constructed to describe factors associated with the outcome of smoking cessation, measured according to the self-reported four-week success rate among 367 outpatient smokers.

RESULTS: ETA was found to be associated with not quitting smoking through the treatment (odds ratio = 0.57; $95 \%$ confidence interval $=0.33-0.96 ; \mathrm{P}<0.05$ ), even after adjustment for dependence level, sociodemographics, nicotine patch use and number of years of smoking.

CONCLUSIONS: Early exposure to nicotine may lead to higher risk of continuing smoking after treatment, in adulthood.
\end{abstract}

\section{INTRODUCTION}

Cigarette smoking, including secondhand smoking, has been identified as the second leading risk factor regarding overall disease burden, only behind high blood pressure. Efforts have been made to control tobacco consumption since the 1970s, such that the overall estimated age-standardized prevalence of daily tobacco smoking declined by $25 \%$ for men and $42 \%$ for women between 1980 and 2012 globally. On the other hand, because of population growth, the number of smokers increased significantly worldwide, from 721 million in 1980 to 967 million in 2012. ${ }^{1}$ A Brazilian study ${ }^{2}$ documented a steep decline in smoking prevalence from $15.6 \%$ in 2006 to $10.8 \%$ in 2014 . Despite this public health success story of reducing smoking, tobacco use continues to adversely influence global health patterns, leading to 5.7 million deaths, and $5.5 \%$ of disability-adjusted life-years (DALYs) in 2010. ${ }^{3,4}$

The health benefits of smoking cessation are well established and it is also known that Brazil presents a relatively high quit ratio, compared with other countries (around $47 \%$ of former daily smokers among those who were ever daily smokers). ${ }^{5}$ Considering this tendency among Brazilian smokers for them to seek to abstain from tobacco use, there is an opportunity to improve existing prevention and treatment strategies that can be seized. It is also known that a majority of smokers would like to stop and indeed attempt to stop many times. However, even though $60 \%-70 \%$ of smokers may intend to stop in any given year, only 3\%-5\% of them remain abstinent through an unassisted attempt, $7 \%-16 \%$ through receiving behavioral therapy and $24 \%$ through receiving behavioral therapy combined with pharmacotherapy. ${ }^{6,7}$

Understanding the determinants of stopping smoking is important for better defining the profile of patients seeking treatment and individuals needing to receive targeted preventive interventions. Nonetheless, there are still few studies on predictors of smoking cessation in the 
general population. A large systematic review found that only the dependence levels were consistently predictive of the success of these attempts. ${ }^{8}$ On the other hand, many studies have tried to elucidate the impact that other factors, such as nicotine replacement therapy, neuropsychiatric comorbidities, smoking more often during the first hours of wakefulness, abstinence symptoms, duration of treatment and early initiation of smoking, have on treatment outcomes. ${ }^{9-11}$ A pre-post field trial study tried to elucidate how an early start to tobacco use impacts treatment outcome and found that there was a positive association between the mean age at which smoking started and cessation rates. This showed that treatment was more likely to provide success when the mean age at which smoking started was greater. ${ }^{12}$

Adolescence is widely recognized as a time of greater risktaking, compared with other age groups. Adolescents are more likely than other developmental age groups to start smoking cigarettes because of normative developmental processes, including heightened reactivity to novel and potentially rewarding stimuli and protracted maturation of cognitive control, along with lower heed given to guidance about the future and heightened sensitivity to peers. ${ }^{13}$ Exposure to nicotine among adolescents has been directly associated with greater risk of later nicotine dependence, ${ }^{13}$ although there is still discussion regarding the evidence of causal effects, as observed in animal models of adolescents, which are more sensitive to nicotine reward effects and less sensitive to their aversive effects. ${ }^{13}$

However, researchers have also considered the presence of shared genetic and environmental risk factors for both dependence and early age of onset. Regarding this issue, a monozygotic co-twin control study ${ }^{14}$ (controlled for genetic and familialenvironmental effects) suggested that early nicotine exposure directly increased the level of later nicotine dependence and craving for cigarettes when unable to smoke, thus supporting the causal effect hypothesis. If the causal effect is true, then reducing early exposure should reduce the risk of subsequent nicotine dependence and all the implications and other disorders to which it predisposes.

\section{OBJECTIVE}

The main objective of the present study was, in the context of a treatment-seeking group of subjects, to test the hypothesis that experimentation with tobacco during adolescence (ETA) is associated with not quitting smoking through treatment, in adulthood, by comparing the smoking cessation rates between two groups of individuals who completed a smoking treatment protocol (one group with and the other without ETA) and adjusting the results for 12 covariates, including the number of years of tobacco smoking, since this may be an important confounder.

\section{METHODS}

\section{Design, ethics and setting}

We conducted a six-week retrospective (historic) cohort study on all the patients who were being treated through a smoking cessation protocol at the Psychosocial Care Center for Alcohol and Drugs in the city of São Caetano do Sul, São Paulo, Brazil, from April 2007 to April 2010. Ethics approval was obtained from the local Institutional Review Board (April 13, 2011; no. 028/2011).

To calculate the sample size, we used the average success rate from smoking cessation treatment that was found among individuals using pharmacotherapy at week 12 in a previous important study (which was around $30 \%) .{ }^{19}$ Considering an alpha of $5 \%$ and an error of $10 \%$, we found that we would need 80.6 individuals enrolled in the exposure subgroups. Fortunately, we had more than 81 individuals in both exposure subgroups (ETA and non-ETA).

\section{Participants}

The subjects were recruited between July 2007 and December 2010 and data were collected using questionnaires applied at the baseline and using a follow-up chart (from T0 to T4; see section 2.1). Patients were recruited through banners that were displayed in the Psychosocial Care Unit (CAPS), and general healthcare professionals in the region were also asked to disseminate the anti-smoking program among smokers in the region. The mean age of the subjects was 51 years. Self-report questionnaires were completed at the baseline with the assistance of healthcare professionals, and evaluations and ratings were recorded at any follow-up appointment.

\section{Treatment protocol}

The treatment protocol included six sessions of weekly group therapy and four consultations with a psychiatrist $(\mathrm{T} 1=0 ; \mathrm{T} 2=$ 1 week; T3 = 3 weeks; T4 = 6 weeks). ${ }^{15-18}$ The group therapy sessions were composed of up to 15 people and were based on the principles of cognitive-behavioral psychotherapy. ${ }^{2}$

\section{Measurements}

In the present study, the cutoff age for starting to use tobacco was taken to be 19 years of age, based on the cutoff used in several recent studies among adolescents ${ }^{20}$ and following data on the age of initiation among Brazilians, ${ }^{2}$ in order to establish the measurement for exposure to tobacco (experimentation with tobacco during adolescence, ETA).

The outcome measurement of success was considered to be four weeks of self-reported abstinence, in accordance with the criteria for "self-reported four-week quitter" that are encompassed in the Russell standard. ${ }^{21}$ Individuals were deemed to have been unsuccessful both if they had not achieved success by the end of the protocol period and if they dropped out. 


\section{Statistical analysis}

The Stata software, version 11, was used to create the database of information from the questionnaires and protocols and to analyze the data. Using the "enter" method, univariate logistic regression and multiple logistic regression were performed. Descriptive analysis comparing ETA and non-ETA groups regarding their sociodemographic and smoking profile was carried out. Then, twelve variables were included in the multiple logistic regression model based on current scientific literature on smoking cessation predictors, as follows: gender; educational level; number of years of smoking; time to first cigarette (i.e. the length of time after waking up until the first cigarette); number of cigarettes per day; occurrence of breathlessness; difficulty in being in non-smoking areas; most difficult cigarette to quit (i.e. the first cigarette in the morning or any other cigarette); tendency to smoke most of the day during sick leave; use of nicotine patch; presence of other smokers at home; and practice of physical activity. The variable "success" was chosen as the dependent variable. These criteria were included in order to analyze the role of ETA in smoking cessation treatment during adulthood, after adjustment for possible confounders.

\section{RESULTS}

Chi-square tests showed significant differences between the ETA and non-ETA groups in relation to the following variables (Table 1): gender $(\mathrm{P}<0.01)$, occurrence of breathlessness $(P<0.05)$, number of years of smoking $(P<0.01)$, age at time of first cigarette $(\mathrm{P}<0.01)$, tendency to smoke most of the day during sick leave $(\mathrm{P}<0.01)$, presence of other smokers at home $(\mathrm{P}<0.05)$ and practice of physical activity $(\mathrm{P}<0.01)$. ETA was reported by $81 \%$ of the men and $67 \%$ of the women; $76 \%$ of those presenting breathlessness; $60 \%$ of those who had been smoking for less than 30 years; $77 \%$ of those who had been smokers for at least 30 years; $77 \%$ of those who had their first cigarette five minutes after waking up; $77 \%$ of those who lived with another smoker; and $78 \%$ of those who did not practice any physical activity. Success was associated with the non-ETA group $(\mathrm{P}<0.05)$, such that $46.6 \%$ of this group were successful, versus $33.7 \%$ in the ETA group.

Table 2 presents the results from the crude and adjusted logistic regression models for treatment success. As shown, ETA was significantly associated with failure of the treatment (odds ratio = $0.57 ; 95 \%$ confidence interval $=0.33-0.96 ; \mathrm{P}<0.05)$. A longer time until the first cigarette and a greater number of years of smoking (duration of smoking) were associated with success. In further crude analysis, duration of smoking (both continuous and categorical) was associated with success. Male gender, low education level, hypertension and shorter time until the first cigarette were associated with the class with greater number of years of smoking, in the adjusted analysis. No multicollinearity was found, i.e. the

Table 1. Descriptive analysis on experimentation with tobacco during adolescence (ETA) and non-ETA groups among 367 smokers who were attending outpatient smoking cessation treatment at a psychosocial care unit (CAPS) in São Caetano, São Paulo, Brazil, 2007-2010

\begin{tabular}{|c|c|c|c|c|c|c|}
\hline \multirow{2}{*}{ Variable } & \multicolumn{2}{|c|}{ Non-ETA } & \multicolumn{2}{|c|}{ ETA } & \multirow{2}{*}{$\chi^{2}$} & \multirow{2}{*}{$\mathbf{P}$} \\
\hline & $\mathbf{n}$ & $\%$ & $\mathbf{n}$ & $\%$ & & \\
\hline \multicolumn{7}{|l|}{ Gender } \\
\hline Female & 79 & 32.6 & 136 & 67.4 & 7.38 & 0.007 \\
\hline Male & 24 & 19.2 & 101 & 80.8 & & \\
\hline \multicolumn{7}{|c|}{ Educational attainment (years of schooling) } \\
\hline Up to 8 years & 45 & 33.8 & 88 & 66.2 & 3.44 & 0.064 \\
\hline 9 or more years & 58 & 24.8 & 176 & 75.2 & & \\
\hline \multicolumn{7}{|l|}{ Breathlessness } \\
\hline No & 49 & 34.3 & 94 & 65.7 & 4.46 & 0.035 \\
\hline Yes & 54 & 24.1 & 170 & 75.9 & & \\
\hline \multicolumn{7}{|c|}{ Number of years smoking } \\
\hline$<30$ & 46 & 39.66 & 70 & 60.34 & 11.2847 & 0.001 \\
\hline$\geq 30$ & 57 & 22.71 & 194 & 77.29 & & \\
\hline \multicolumn{7}{|l|}{ Cigarettes per day } \\
\hline$<30$ & 71 & 31.7 & 153 & 68.3 & 3.7543 & 0.053 \\
\hline$\geq 30$ & 32 & 22.38 & 111 & 77.62 & & \\
\hline \multicolumn{7}{|c|}{ Time to first cigarette in the morning } \\
\hline$\leq 5$ minutes & 50 & 22.83 & 169 & 77.17 & 7.3699 & 0.007 \\
\hline$>5$ minutes & 53 & 35.81 & 95 & 64.19 & & \\
\hline \multicolumn{7}{|c|}{ Difficulty staying in non-smoking areas } \\
\hline No & 51 & $32 ., 9$ & 104 & 67.1 & 3.0139 & 0.083 \\
\hline Yes & 52 & 24.64 & 159 & 75.36 & & \\
\hline
\end{tabular}

Most difficult cigarette to quit

First cigarette in the morning

Any other

cigarette

$\begin{array}{llll}31 & 25.2 & 92 & 74.8\end{array}$

Tendency to smoke during a sick period

$\begin{array}{lllllll}\text { No } & 50 & 41.32 & 71 & 58.68 & 15.1435 & <0.01 \\ \text { Yes } & 52 & 21.76 & 187 & 78.24 & & \end{array}$

Other smokers at home

$\begin{array}{lllllll}\text { No } & 56 & 34.36 & 107 & 65.64 & 5.6111 & \mathbf{0 . 0 1 8}\end{array}$

$\begin{array}{lllll}\text { Yes } & 47 & 23.15 & 156 & 76.85\end{array}$

Physical activity

No

$\begin{array}{llll}46 & 22.22 & 161 & 77.78\end{array}$

7.3075

0.007

Yes

Nicotine patch

No

55

Yes

upropion

No

Yes

Nortriptyline

No

Yes

18.18

$03 \quad 102 \quad 64.97$

64.97

Nicotine gum

No

Yes

Success

No

Yes

Total

$\begin{array}{llll}99 & 28.78 & 245 & 71.22\end{array}$

1.1483

0.284

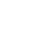

$\begin{array}{llll}55 & 28.35 & 139 & 71.65\end{array}$

0.0166

0.898

$\begin{array}{llll}48 & 27.75 & 125 & 72.25\end{array}$

$\begin{array}{lllllll}100 & 28.99 & 245 & 71.01 & 2.1152 & 0.146\end{array}$

$\begin{array}{llll}3 & 14.29 & 18 & 85.71\end{array}$

(1)

(1)


Table 2. Results from multiple logistic regression model regarding success in smoking cessation among 367 smokers who were attending outpatient smoking cessation treatment at a psychosocial care unit (CAPS) in São Caetano, São Paulo, Brazil, 2007-2010

\begin{tabular}{|c|c|c|c|c|c|c|c|c|}
\hline Variables & $\mathrm{n}$ & $\%$ & OR & aOR & {$[95 \%$} & $\mathrm{Cl}]$ & $\mathbf{P}$ & VIF \\
\hline \multicolumn{9}{|l|}{ Exposure } \\
\hline Non-ETA & 48 & 46.6 & 1.00 & 1.00 & & & & \\
\hline ETA & 89 & 33.7 & 0.58 & 0.57 & 0.33 & 0.96 & 0.037 & 1.17 \\
\hline \multicolumn{9}{|l|}{ Covariates } \\
\hline \multicolumn{9}{|l|}{ Gender } \\
\hline Female & 96 & 39.7 & 1.00 & 1.00 & & & & \\
\hline Up to 8 years & 58 & 43.6 & 1.00 & 1.00 & & & & \\
\hline 9 or more years & 79 & 33.8 & 0.65 & 0.68 & 0.42 & 1.09 & 0.108 & 1.05 \\
\hline \multicolumn{9}{|l|}{ Number of years smoking } \\
\hline Less than 30 years & 34 & 29.3 & 1.00 & 1.00 & & & & \\
\hline 30 years or more & 103 & 41.0 & 1.67 & 1.93 & 1.15 & 3.25 & 0.014 & 1.08 \\
\hline \multicolumn{9}{|l|}{ Cigarettes per day } \\
\hline Less than 30 & 91 & 40.6 & 1.00 & 1.00 & & & & \\
\hline 30 or more & 46 & 32.2 & 0.69 & 0.92 & 0.55 & 1.54 & 0.748 & 1.22 \\
\hline \multicolumn{9}{|l|}{ Breathlessness } \\
\hline No & 50 & 35.0 & 1.00 & 1.00 & & & & \\
\hline Yes & 87 & 38.8 & 1.18 & 1.57 & 0.95 & 2.60 & 0.077 & 1.13 \\
\hline \multicolumn{9}{|c|}{ Difficulty being in non-smoking areas } \\
\hline No & 64 & 41.3 & 1.00 & 1.00 & & & & \\
\hline Yes & 72 & 34.1 & 0.73 & 0.81 & 0.49 & 1.35 & 0.425 & 1.26 \\
\hline \multicolumn{9}{|l|}{ Most difficult cigarette to quit } \\
\hline Yes & 133 & 38.7 & 2.83 & 2.42 & 0.72 & 8.08 & 0.152 & 1.10 \\
\hline \multicolumn{9}{|l|}{ Other smokers at home } \\
\hline No & 58 & 35.6 & 1.00 & 1.00 & & & & \\
\hline Yes & 79 & 38.9 & 1.15 & 1.16 & 0.72 & 1.86 & 0.535 & 1.08 \\
\hline \multicolumn{9}{|l|}{ Physical activity } \\
\hline No & 75 & 36.2 & 1.00 & 1.00 & & & & \\
\hline Yes & 62 & 39.5 & 1.14 & 1.08 & 0.67 & 1.75 & 0.755 & 1.13 \\
\hline
\end{tabular}

Non-success was the reference category; $\mathrm{aOR}=$ adjusted odds ratio; $\mathrm{Cl}=$ confidence interval; $\mathrm{VIF}=$ variance inflation factor; $\mathrm{ETA}=$ experimentation with tobacco during adolescence.

variance inflation factor (VIF) values were very low. No significant difference was found between the ETA and non-ETA groups regarding treatment drop-out rate $(55.9 \%$ versus $48.5 \%$, respectively). A further crude and adjusted logistic regression was carried out excluding those who dropped out. Success continued to be associated with the non-ETA group $(\mathrm{P}<0.05)$, which had a success rate of $90.6 \%$, versus $76.7 \%$ in the ETA group.

\section{DISCUSSION}

Our study shows that experimentation with tobacco during adolescence is an influential factor regarding failure of smoking cessation, independently of the level of dependence, number of years of smoking and other potential confounders. Consistent with the available literature, ${ }^{14,22}$ our results confirmed that experimentation with tobacco during adolescence was also associated 
with greater severity of smoking history, such as having been a smoker for longer and reporting occurrences of the symptom of breathlessness. Since the risk of developing tobacco-related disease increases as a function of the duration of time for which tobacco is used, adolescent users are at particularly high risk of physical consequences from tobacco use later on.

Tobacco smoking among adolescents remains a persistent threat to public health, ${ }^{1}$ and most adolescents who use tobacco regularly (e.g. monthly or more frequently) continue their use into adulthood. For example, while only $5 \%$ of adolescent smokers see themselves as continuing to smoke five years later, $75 \%$ are actually still smoking eight years later. ${ }^{23}$

Defining the boundaries of this period and what it encompasses is a matter of controversy. A cross-sectional study revealed differences in brain activation between late adolescents (18-19 years old) and young adults (23-25 years old) during cognitive control, thus indicating that protracted functional development of the cortex continues into these individuals' twenties. ${ }^{24}$ Moreover, maturational changes in active synaptic pruning, a process that is thought to enhance information processing capacity and speed and information rearrangement, and in white matter myelination, a process that aids the functional integration of widely distributed circuitry, begin to accelerate during early adolescence and reach a plateau approximately at the ages of 24-25 years. ${ }^{25}$

It is well known that adolescence is a crucial period for the development of cognitive and executive functions, working memory, reward processing, emotional regulation and motivated behavior. There is greater vulnerability to the effects of nicotine during this period. More specifically, the neurobiological impact of earlyonset smoking may be especially deleterious because maturational changes in active synaptic pruning and rearrangement and white matter myelination begin to accelerate during early adolescence. ${ }^{26}$ Persistent exposure to nicotine via tobacco smoking throughout adolescent neurodevelopment can damage newly maturing synaptic signaling pathways and alter the patterns of neurotransmitter release, thereby increasing sensitivity to reward-related neural activation and susceptibility towards developing nicotine dependence. ${ }^{27}$ One recent laboratory study ${ }^{28}$ demonstrated that cigarette craving, as assessed through the statement "Nothing would be better than smoking a cigarette right now", was greater among early-onset smokers than it was among late-onset smokers and healthy non-smokers. Through use of electroencephalography and event-related potentials, this study ${ }^{28}$ showed elevated reactivity in the early-onset group after these individuals viewed salient smoking-related images.

Positive expectancies develop as a result of heightened sensitivity to rewards during this period and often when there is a pleasurable initial smoking experience. However, there are also particular risk factors to be addressed regarding the easy availability of cigarettes and the positive social norms regarding smoking. ${ }^{13}$ The extent to which cigarette availability (perhaps concomitant to exposure to peers) affects reward-related processing among adolescent smokers has not been fully examined. This remains an important target for future research. Nonetheless, the findings of the present study add strength to policies that aim to restrict adolescents' access to tobacco.

The finding that greater numbers of years of smoking was associated with success in the treatment was conflictive with some opposite findings in the recent literature. ${ }^{29}$ However, it is important to note that the evidence from the previous study came from a general population sample, which differed from the type of sample of the present study (clinical). In addition, more than $70 \%$ of the subjects included in the present study were undergoing psychiatric treatment for mental disorders other than nicotine dependence. Such populations are known to have exceptionally high smoking rates, due to biological, psychological and social factors. Moreover, adding to previous studies that demonstrated how starting to smoke early on acts as a negative predictor for cessation success, ${ }^{9,12}$ the present study also reaffirmed the importance of this variable in the specific population of individuals with mental health and addiction disorders.

The present study had limitations, especially with regard to subjective measurement of success, given that the majority of the variables were based on the patients' self-reports. Objective data, such as quantification of salivary cotinine and carbon monoxide, could have been useful. ${ }^{30}$ Despite the evidence that self-reported data regarding cessation of tobacco use is reliable ${ }^{29}$ biological data such as assessment of the quantities of salivary cotinine and carbon monoxide could have been useful. However, there is evidence showing that self-reported data on cessation of tobacco use is reliable. ${ }^{31}$ Presentation of severe unpleasant withdrawal symptoms during the first days after quitting and the consequent higher likelihood of relapse emphasize the relevance of maintaining abstinence during this period in order to achieve long-term cessation. In middleincome countries, studies conducted on anti-smoking treatment in community settings have lacked instruments to objectively quantify nicotine abstinence and, thus, have continued to use self-report measurements. ${ }^{10,32-34}$ Others limitations that need to be added were that the number of patients excluded was not recorded according to cause and that there was a high drop-out rate.

Our results may add clinical considerations to the neurobiological evidence available in relation to experimentation with tobacco during adolescence. ${ }^{14}$ One particular feature of the treatment protocol that was used at this unit was that cognitive-behavioral therapy in groups was provided, conducted by a psychologist with broad experience relating to addiction. ${ }^{20}$ This type of experience differs from the supportive counselling that is provided by general practitioners in most smoking cessation clinics. 


\section{CONCLUSIONS}

Early exposure to nicotine may lead to higher risk of continuing smoking after treatment in adulthood. This finding needs to be incorporated into prevention and treatment strategies, in order to enhance health literacy regarding severe modifiable risk factors within smoking.

\section{REFERENCES}

1. Ng M, Freeman MK, Fleming TD, et al. Smoking prevalence and cigarette consumption in 187 countries, 1980-2012. Jama. 2014;311(2):183-92. PMID: 24399557; doi: 10.1001/jama.2013.284692.

2. Monteiro CA, Cavalcante TM, Moura EC, Claro RM, Szwarcwald CL. Population-based evidence of a strong decline in the prevalence of smokers in Brazil (1989-2003). Bull World Health Organ. 2007;85(7):52734. PMID: 17768501; doi: 10.2471/BLT.06.039073.

3. Lim SS, Vos T, Flaxman AD, et al. A comparative risk assessment of burden of disease and injury attributable to 67 risk factors and risk factor clusters in 21 regions, 1990-2010: a systematic analysis for the Global Burden of Disease Study 2010. Lancet. 2012;380(9859):2224-60. PMID: 23245609; doi: 10.1016/S0140-6736(12)61766-8.

4. GBD 2010 change in leading causes and risks between 1990 and 2010 [Internet]. Institute for Health Metrics and Evaluation. 2013.

5. Giovino GA, Mirza SA, Samet JM, et al. Tobacco use in 3 billion individuals from 16 countries: an analysis of nationally representative cross-sectional household surveys. Lancet. 2012;380(9842):668-79. PMID: 22901888; doi: 10.1016/S0140-6736(12)61085-X.

6. Laniado-Laborin R. Smoking cessation intervention: an evidence-based approach. Postgrad Med. 2010;122(2):74-82. PMID: 20203458; doi: 10.3810/pgm.2010.03.2124.

7. Zhu S, Melcer T, Sun J, Rosbrook B, Pierce JP. Smoking cessation with and without assistance: a population-based analysis. Am J Prev Med. 2000;18(4):305-11. PMID: 10788733; doi: 10.1016/S07493797(00)00124-0.

8. Vangeli E, Stapleton J, Smit ES, Borland R, West R. Predictors of attempts to stop smoking and their success in adult general population samples: a systematic review. Addiction. 2011;106(12):2110-21. PMID: 21752135; doi: 10.1111/j.1360-0443.2011.03565.x.

9. West R, McEwen A, Bolling K, Owen L. Smoking cessation and smoking patterns in the general population: a 1-year follow-up. Addiction. 2001;96(6):891-902. PMID: 11399220; doi: 10.1080/09652140020051013.

10. França $S A$, Neves $A L$, Souza $T A$, et al. Fatores associados à cessação do tabagismo [Factors associated with smoking cessation]. Rev Saúde Pública. 2015;49:10. PMID: 25741649; doi: 10.1590/S00348910.2015049004946.

11. Prado GF, Lombardi EM, Bussacos MA, et al. A real-life study of the effectiveness of different pharmacological approaches to the treatment of smoking cessation: re-discussing the predictors of success. Clinics (Sao Paulo). 2011;66(1):65-71. PMID: 21437438; doi: 10.1590/S180759322011000100012.
12. Abdolahinia A, Sadr M, Hessami Z. Correlation between the age of smoking initiation and maintaining continuous abstinence for 5 years after quitting. Acta Med Iran. 2012;50(11):755-9. PMID: 23292627.

13. Lydon DM, Wilson SJ, Child A, Geier CF. Adolescent brain maturation and smoking: what we know and where we're headed. Neurosci Biobehav Rev. 2014;45:323-42. PMID: 25025658; doi: 10.1016/j. neubiorev.2014.07.003.

14. Kendler KS, Myers J, Damaj MI, Chen X. Early smoking onset and risk for subsequent nicotine dependence: a monozygotic co-twin control study. Am J Psychiatry. 2013;170(4):408-13. PMID: 23318372; doi: 10.1176/ appi.ajp.2012.12030321

15. Loreto AR, Carvalho CFC, Frallonardo FP, et al. Smoking Cessation Treatment for Patients With Mental Disorders Using CBT and Combined Pharmacotherapy. J Dual Diagn. 2017;13(4):238-46. PMID: 28489501; doi: 10.1080/15504263.2017.1328149.

16. Castaldelli-Maia JM, Loreto AR, Guimarães-Pereira BBS, et al. Smoking cessation treatment outcomes among people with and without mental and substance use disorders: An observational real-world study. Eur Psychiatry. 2018;52:22-28. PMID: 29609056; doi: 10.1016/j. eurpsy.2018.02.005.

17. Castaldelli-Maia JM, Carvalho CF, Armentano F, et al. Outcome predictors of smoking cessation treatment provided by an addiction care unit between 2007 and 2010. Brazilian J Psychiatry. 2013;35(4):338-46. PMID: 24402207; doi: 10.1590/1516-4446-2012-0907.

18. Castaldelli-Maia JM, Loreto AR, Carvalho CF, Frallonardo FP, de Andrade AG. Retention predictors of a smoking treatment provided by a public psychosocial unit in Brazil. Int Rev Psychiatry. 2014;26(4):515-23. PMID: 25137119; doi: 10.3109/09540261.2014.928272.

19. Anthenelli RM, Benowitz NL, West R, et al. Neuropsychiatric safety and efficacy of varenicline, bupropion, and nicotine patch in smokers with and without psychiatric disorders (EAGLES): a double-blind, randomised, placebo-controlled clinical trial. Lancet. 2016;387(10037):2507-20. PMID: 27116918; doi: 10.1016/S0140-6736(16)30272-0.

20. Freedman KS, Nelson NM, Feldman LL. Smoking initiation among young adults in the United States and Canada, 1998-2010: a systematic review. Prev Chronic Dis. 2012;9:E05. PMID: 22172172; doi: 10.5888/ pcd9.110037.

21. West R. Assessing smoking cessation performance in NHS Stop Smoking Services: The Russell Standard (Clinical). Internet [2005]. Available from: http://wwwncsctcouk/usr/pub/assessing-smoking-cessationperformance-in-nhs-stop-smoking-services-the-russell-standardclinicalpdf. Accessed in 2018 (May 25).

22. Park SM, Son KY, Lee YJ, et al. A preliminary investigation of early smoking initiation and nicotine dependence in Korean adults. Drug Alcohol Depend. 2004;74(2):197-203. PMID: 15099663; doi: 10.1016/j. drugalcdep.2004.01.001.

23. Sussman S, Dent CW, Severson H, Burton D, Flay BR. Self-initiated quitting among adolescent smokers. Prev Med. 1998;27(5 Pt 3):A19-28. PMID: 9808814; doi: 10.1006/pmed.1998.0379. 
24. Veroude K, Jolles J, Croiset G, Krabbendam L. Changes in neural mechanisms of cognitive control during the transition from late adolescence to young adulthood. Dev Cogn Neurosci. 2013;5:63-70. PMID: 23376475; doi: 10.1016/j.den.2012.12.002.

25. Tamnes CK, Ostby $Y$, Walhovd KB, et al. Neuroanatomical correlates of executive functions in children and adolescents: a magnetic resonance imaging (MRI) study of cortical thickness. Neuropsychologia. 2010;48(9):2496-508. PMID: 20434470; doi: 10.1016/j. neuropsychologia.2010.04.024.

26. Paus T, Zijdenbos A, Worsley K, et al. Structural maturation of neural pathways in children and adolescents: in vivo study. Science. 1999;283(5409):1908-11. PMID: 10082463; doi: 10.1126/ science.283.5409.1908.

27. Dwyer JB, McQuown SC, Leslie FM. The dynamic effects of nicotine on the developing brain. Pharmacol Ther. 2009;122(2):125-39. PMID: 19268688; doi: 10.1016/j.pharmthera.2009.02.003.

28. Mashhoon Y, Betts J, Farmer SL, Lukas SE. Early onset cigarette smokers exhibit greater P300 reactivity to smoking-related stimuli and report greater craving. Brain Res. 2018;1687:173-84. PMID: 29524436; doi: 10.1016/j.brainres.2018.02.037.

29. Holm M, Schioler L, Andersson E, et al. Predictors of smoking cessation: A longitudinal study in a large cohort of smokers. Respir Med. 2017;132:164-9. PMID: 29229092; doi: 10.1016/j.rmed.2017.10.013.

30. Aveyard P, Bauld L. Incentives for promoting smoking cessation: What we still do not know. The Cochrane database of systematic reviews. 2011;Apr13(8):ED000027. PMID: 21833985; doi: 10.1002/14651858. ED000027.

31. Popova L, Ling PM. Alternative tobacco product use and smoking cessation: a national study. Am J Public Health. 2013;103(5):923-30. PMID: 23488521; doi: 10.2105/AJPH.2012.301070

32. Castaldelli-Maia JM, Martins SS, Walker N. The effectiveness of Cytisine versus Nicotine Replacement Treatment for smoking cessation in the Russian Federation. Int J Drug Policy. 2018;58:121-5. PMID: 29929154; doi: 10.1016/j.drugpo.2018.05.012.

33. Castaldelli-Maia JM, Martins SS, Storr CL, et al. Investigating the dimensional diagnosis of ICD-11 nicotine dependence. Psychol Addict Behav. 2018;32(4):415-25. PMID: 29927280; doi: 10.1037/adb0000369.

34. Castaldelli-Maia JM, Andrade LH, Storr CL, et al. The latent trait of ICD-11 nicotine dependence criteria: Dimensional and categorical phenotypes. Psychiatry Res. 2018;266:275-83. PMID: 29605101; doi: 10.1016/j.psychres.2018.03.018.
Date of first submission: May 21, 2018

Last received: March 1, 2019

Accepted: March 14, 2019

\section{Address for correspondence:}

João Mauricio Castaldelli-Maia

Centro de Estudos em Saúde Mental do ABC, Departamento de Neurociência, Faculdade de Medicina do ABC (FMABC), Fundação ABC Avenida Lauro Gomes, 2.000

Santo André (SP) — Brasil

CEP 09060-870

Tel. (+55 11) 7897-8948

E-mail:jmcmaia2@gmail.com

Conflict of interest: None

Sources of funding: None 